\title{
NOTE ON AN IRREGULAR EXPANSION PROBLEM*
}

\author{
BY DUNHAM JACKSON
}

One of the simplest expansion problems depending on a set of boundary conditions of the type which Birkhoff has called irregular, is that associated with the system

$$
\begin{gathered}
\frac{d^{3} u}{d x^{3}}+\rho^{3} u=0, \\
u(0)=0, \quad u^{\prime}(0)=0, \quad u(\pi)=0 .
\end{gathered}
$$

Let $u_{1}(x), u_{2}(x), \cdots$ be the characteristic functions of this differential system. The writer showed, a number of years ago, $\dagger$ that the formal expansion of an arbitrary continuous function $f(x)$ in a series of the form

$$
a_{1} u_{1}(x)+a_{2} u_{2}(x)+\cdots
$$

will ordinarily be divergent, even if $f(x)$ satisfies conditions which would insure the convergence of its development in terms of the characteristic functions of a regular system; and it may diverge even if $f(x)$ is analytic throughout the interval $(0, \pi)$. A subsequent paper by Hopkins $\ddagger$ specified a restricted class of analytic functions to which $f(x)$ must belong, if the expansion is to converge uniformly to the desired value. He showed that the necessary condition thus obtained, if supplemented by certain secondary hypotheses, is also sufficient. He did not discuss the question whether the formal expansion, if uniformly convergent at all, must necessarily have $f(x)$ for its sum. His analysis, however, makes it possible to recognize without difficulty that this is the case. It is

* Presented to the Society, April 23, 1921.

$\dagger$ D. Jackson, Expansion problems with irregular boundary conditions, Proceedings of the American Academy, vol. 51 (1915-16), pp. 383417; see pp. 384-393.

$\ddagger$ J. W. Hopkins, Some convergent developments associated with irregular boundary conditions, Transactions of this Society, vol. 20 (1919), pp. 245-259. 
the purpose of the following paragraphs to supply the details of the demonstration.*

The system adjoint to (1) is

$$
\begin{gathered}
\frac{d^{3} v}{d x^{3}}-\rho^{3} v=0, \\
v(0)=0, \quad v(\pi)=0, \quad v^{\prime}(\pi)=0 .
\end{gathered}
$$

Its characteristic functions may be denoted by $v_{1}(x), v_{2}(x), \cdots$, and then the coefficients in the series (2) will be given by

$$
a_{n}=\frac{1}{q_{n}} \int_{0}^{\pi} f(x) v_{n}(x) d x, \quad q_{n}=\int_{0}^{\pi} u_{n}(x) v_{n}(x) d x .
$$

If the series converges uniformly, its sum $g(x)$ will be a continuous function vanishing for $x=0$ and for $x=\pi$ (and satisfying also the condition $g^{\prime}(0)=0$, though it is not necessary to insist on that point here). By the orthogonal properties of the system of functions $u_{n}(x), v_{n}(x)$, we see that

$$
\frac{1}{q_{n}} \int_{0}^{\pi} g(x) v_{n}(x) d x=a_{n},
$$

so that the difference

$$
r(x)=f(x)-g(x)
$$

has the property that

$$
\int_{0}^{\pi} r(x) v_{n}(x) d x=0 \quad(n=1,2, \cdots) .
$$

We shall assume at first that $f(x)$ vanishes at both ends of the interval $(0, \pi)$, and then $r(x)$ will have this property also. It is to be shown that $r(x)$ must vanish identically.

It follows from Hopkins's work $\nmid$ that the formal develop-

* This proof is more or less in line with a conversation between Professor Birkhoff and the writer, which took place early in 1917, before Mr. Hopkins's work was completed. Neither party to the conversation can remember definitely now what was said, except that it probably covered a part, but certainly not the whole, of what is done here. The fundamental idea of the proof has been used frequently in other connections.

$\dagger$ Loc.cit., Theorem III, and p. 259, footnote. The proof can also be based directly on $\S 3$ of the paper, without reference to the more general method of $\S 4$. 
ment of

$$
x^{3 k+2}-\pi^{3 k} x^{2}
$$

in a series of the form (2) converges uniformly for $0 \leqq x \leqq \pi$, if $k$ is any positive integer. Since the system (1) and the system (3) are carried over into each other by the substitution of $\pi-x$ for $x$, and, in particular*

it follows that

$$
v_{n}(x)=u_{n}(\pi-x)
$$

$$
p_{k}(x)=(\pi-x)^{3 k+2}-\pi^{3 k}(\pi-x)^{2}
$$

can be expanded in a uniformly convergent series of the functions $v_{n}(x)$.

It will be shown that $r(x)$ can be approximately represented, uniformly for $0 \leqq x \leqq \pi$, with any desired degree of accuracy, by means of a linear combination of a finite number of the expressions $p_{k}(x)$. Hence $r(x)$ can be similarly represented in terms of the functions $v_{n}(x)$, each $p_{k}(x)$ being replaced by a sufficient number of terms of its expansion in series of the $v$ 's.

The hypothesis has already provided that $r(0)=r(\pi)=0$. If $r_{1}(x)$ is defined as equal to $r(x)$ for $0 \leqq x \leqq \pi-2 \delta$, equal to $(\pi-x)^{2}$ for $\pi-\delta \leqq x \leqq \pi$, and linear and continuous for $\pi-2 \delta \leqq x \leqq \pi-\delta$, then $r_{1}(x)$ will differ arbitrarily little from $r(x)$ throughout $(0, \pi)$, if $\delta$ is taken sufficiently small, and will have the form

$$
r_{1}(x)=(\pi-x)^{2} q(x),
$$

where $q(x)$ is continuous for $0 \leqq x \leqq \pi$, and vanishes for $x=0$. The quantities involved being real, $\pi-x$ is a singlevalued continuous function of $(\pi-x)^{3}$, and the same is true of $x$. Hence $q(x)$ is a single-valued continuous function of $(\pi-x)^{3}$ for $0 \leqq x \leqq \pi$. Therefore by Weierstrass's theorem, it can be approximately represented with any desired accuracy by a polynomial in $(\pi-x)^{3}$. That is, if $\epsilon$ is any positive quantity, there will exist an exponent $m$ and a set of coefficients

\footnotetext{
* Cf., e.g., D. Jackson, loc. cit., pp. 387, 389.
} 
$A_{0}, A_{1}, \cdots, A_{m}$, so that

$$
\begin{aligned}
\mid A_{0}+A_{1}(\pi-x)^{3}+A_{2}(\pi-x)^{6} & +\cdots \\
& +A_{m}(\pi-x)^{3 m}-q(x) \mid<\epsilon
\end{aligned}
$$

throughout $(0, \pi)$. In particular, for $x=0$, since $q(0)=0$,

$$
\left|A_{0}+A_{1} \pi^{3}+A_{2} \pi^{6}+\cdots+A_{m} \pi^{3 m}\right|<\epsilon .
$$

Hence, by subtraction within the bars,

$$
\begin{aligned}
\mid A_{1}\left[(\pi-x)^{3}-\pi^{3}\right]+ & \cdots \\
& +A_{m}\left[(\pi-x)^{3 m}-\pi^{3 m}\right]-q(x) \mid<2 \epsilon ;
\end{aligned}
$$

from (5) and (6),

$$
\left|A_{1} p_{1}(x)+\cdots+A_{m} p_{m}(x)-r_{1}(x)\right|<2 \pi^{2} \epsilon ;
$$

and the existence of the desired approximation for $r(x)$ can be inferred at once.

It is to be regarded as established, then, that $r(x)$ can be represented with any required accuracy by a linear combination of the $v$ 's, or, what amounts to the same thing, that $r(x)$ can be expanded in a uniformly convergent series of the form

$$
r(x)=C_{1} V_{1}(x)+C_{2} V_{2}(x)+\cdots,
$$

where $V_{n}(x)$ is a linear combination of $v_{1}(x), v_{2}(x), \cdots, v_{n}(x)$, with constant coefficients. Because of (4),

$$
\int_{0}^{\pi} r(x) V_{n}(x) d x=0 \quad(n=1,2, \cdots) .
$$

Consequently

$$
\int_{0}^{\pi}[r(x)]^{2} d x=\int_{0}^{\pi} r(x)\left[C_{1} V_{1}(x)+C_{2} V_{2}(x)+\cdots\right] d x=0,
$$

and $r(x)$, being continuous, must vanish identically.

It has been assumed hitherto that $f(x)$ vanishes at the ends of the interval. This must in fact be the case, if $f(x)$, always supposed continuous, is to give rise to a uniformly convergent expansion. Let us make the contrary hypothesis, and show that it leads to a contradiction. The continuous function $g(x)$ must still vanish at 0 and $\pi$, and $r(x)$ will have the same 
value as $f(x)$ at these points, being accordingly different from zero at one or both of them. It will then be no longer possible to approach $r(x)$ uniformly by means of linear combinations of the $v$ 's. If $\epsilon$ is any positive quantity, however, it will be possible to choose a function $r_{2}(x)$, continuous throughout the interval and vanishing at 0 and $\pi$, in such a way that

$$
\int_{0}^{\pi}\left|r(x)-r_{2}(x)\right| d x<\frac{\epsilon}{2}
$$

Then $r_{2}(x)$, by the reasoning applied to $r(x)$ under the previous hypothesis, can be approached by a linear combination of the $v$ 's, from $v_{1}$ to $v_{n}$, say, with a maximum error not exceeding $\epsilon /(2 \pi)$. If the approximating function is denoted by $W_{n}(x)$ we shall have

$$
\int_{0}^{\pi}\left|r(x)-W_{n}(x)\right| d x<\epsilon .
$$

If we do this for a succession of values of $\epsilon$ approaching zero, we obtain a succession of functions* $W_{n}(x)$, such that

$$
\lim _{n \rightarrow \infty} \int_{0}^{\pi}\left|r(x)-W_{n}(x)\right| d x=0 .
$$

Let $M$ be the maximum of $|r(x)|$; then

$$
\left|\int_{0}^{\pi} r(x)\left[r(x)-W_{n}(x)\right] d x\right| \leqq M \int_{0}^{\pi}\left|r(x)-W_{n}(x)\right| d x,
$$

so that

$$
\lim _{n \rightarrow \infty} \int_{0}^{\pi} r(x)\left[r(x)-W_{n}(x)\right] d x=0 .
$$

Since $\int r W_{n} d x=0$ for all values of $n$, it is seen again that $\int r^{2} d x=0$, and $r(x)$ vanishes identically. This is at the same time a verification of the principal result to be established, and a proof that the hypothesis with regard to the non-vanishing of $f(x)$ at the ends of the interval was inadmissible.

The University of Minnesota.

* It is allowable to think of the index $n$ as taking on all positive integral values, since a linear combination of the first $n$ of the $v$ 's is at the same time a linear combination of the first $n^{\prime}$ of them, if $n^{\prime}$ is any number greater than $n$. 